\title{
Fitossociologia, caracterização sucessional e síndromes de dispersão da comunidade arbórea de remanescente urbano de Floresta Estacional Semidecidual em Monte Carmelo, Minas Gerais
}

Phytossociology, successional groups and dispersal syndromes of the tree community in an urban remnant of semideciduous forest in Monte Carmelo, Minas Gerais

Jamir Afonso do Prado Júnior ${ }^{1,5}$, Sérgio de Faria Lopes ${ }^{2}$, Ivan Schiavini ${ }^{3}$, Vagner Santiago do Vale ${ }^{2}$, Ana Paula de Oliveira ${ }^{2}$, André Eduardo Gusson ${ }^{2}$, Olavo Custódio Dias Neto ${ }^{2} \&$ Manuela Stein ${ }^{4}$

\begin{abstract}
Resumo
Os parques urbanos têm uma importância estratégica para a qualidade de vida dos centros urbanos, contribuindo com importantes serviços ambientais e sociais. O conhecimento da estrutura florestal tem ajudado a subsidiar a conservação destes parques. A partir da análise fitossociológica das espécies arbóreas de um fragmento de Floresta Estacional Semidecidual (120 ha), este estudo classificou o estado de conservação deste remanescente. Foram amostrados todos os indivíduos arbóreos com CAP $\geq 15 \mathrm{~cm}$, presentes em 25 parcelas permanentes $(20 \times 20 \mathrm{~m})$ e as espécies foram classificadas por grupos sucessionais e síndrome de dispersão. Foram registrados 798 indivíduos distribuídos em 98 espécies, pertencentes a 37 famílias. As espécies secundárias iniciais apresentaram maiores valores na riqueza de espécies, densidade e valor de importância, seguido das espécies secundárias tardias que apresentaram maior dominância e das espécies pioneiras que tiveram pouca representatividade. Quanto à síndrome de dispersão, mais de 77\% da comunidade é representada por indivíduos zoocóricos. Tais resultados aumentam a valoração do Parque da Matinha reafirmando a importância deste parque urbano na conservação da fauna e flora nativa, e reforçam a necessidade de criar políticas públicas para utilização do parque pelos visitantes.
\end{abstract}

Palavras-chave: grupos ecológicos, parque urbano, síndrome de dispersão, sucessão florestal.

\begin{abstract}
Urban parks have a strategic importance fot the quality of life in urban centers, contributing to significant environmental and social services. Knowledge of forest structure has helped to subsidize the maintenance of these parks. Based on the phytosociological analysis of tree species in a semideciduous forest fragment (120 ha), this study assessed the conservation status of this remnant. We sampled all trees with $\mathrm{CBH} \geq$ $15 \mathrm{~cm}$, present in 25 permanent plots $(20 \times 20 \mathrm{~m})$, and classified the species as to successional groups and dispersal syndromes. We recorded 798 individuals in 98 species belonging to 37 families. Early secondary species had the highest values of species richness, density and importance value, followed by late secondary species with the highest dominance, and pioneer species with low representation. Regarding the dispersal syndrome, over $77 \%$ of the community is represented by species with zoochoric dispersal. These results increase the valuation of the "Parque da Matinha", reaffirming the importance of this urban park for the conservation of native fauna and flora, and supporting the need for public policies of park use by visitors.
\end{abstract}

Key words: ecological groups, urban park, dispersal syndrome, forest succession.

\footnotetext{
${ }^{1}$ Curso de Pós graduação em Biologia Vegetal (Universidade Federal de Uberlândia)

${ }^{2}$ Curso de Pós graduação em Ecologia e Conservação de Recursos Naturais (Universidade Federal de Uberlândia)

${ }^{3}$ Professor Doutor do Instituto de Biologia, Universidade Federal de Uberlândia

${ }^{4}$ Bióloga graduada na Universidade Federal de Uberlândia, MG, Brasil

${ }^{5}$ Autor para correspondência: jamirjunior@yahoo.com.br
} 


\section{Introdução}

A conservação da biodiversidade representa um dos maiores desafios deste século, em função do elevado nível de perturbações antrópicas nos ecossistemas naturais (Pimm et al. 2001). A crescente fragmentação das comunidades florestais, que reduz as áreas naturais numa taxa de 1,2\% ao ano (Galleti et al. 2003), altera a capacidade de auto-manutenção destes ecossistemas. Dos remanescentes, muitos estão representados por fragmentos pequenos, imersos numa paisagem dominada pela agricultura e pelos centros urbanos (Durigan et al. 2000). Considerando que urbanização e agropecuária geram grande riqueza para o país, torna-se ainda mais difícil equilibrar o crescimento econômico com a conservação das áreas de vegetação restantes (Aquino \& Miranda 2008).

Nesse contexto, os parques urbanos e espaços verdes têm uma importância estratégica para a qualidade de vida da crescente sociedade cada vez mais urbanizada. Estudos indicam que a presença de recursos naturais (parques urbanos e florestas) e de seus componentes (árvores e corpos d'água) em centros urbanos contribui substancialmente para a qualidade de vida da população (Chiesura 2004). Além de importantes serviços ambientais como purificação do ar e da água, filtragem de vento e de ruídos e estabilização microclimática (Dwyer et al. 1992; Nowak et al. 2006), as áreas naturais prestam serviços sociais e psicológicos de importância crucial para a habitabilidade das cidades modernas e o bem-estar dos moradores urbanos (Ulrich 1981; Hartig et al. 1991; Conway 2000). Em sintonia a estes benefícios, também tem sido comprovada a valoração econômica dos parques urbanos, seja na redução dos gastos com o controle da poluição e economia de energia das construções, seja no aumento da atratividade da cidade e consequente geração de empregos e receitas (Tagtow 1990; Coley et al. 1997; Sander et al. 2010).

Os parques urbanos constantemente estão sob interferência da população e a ausência de políticas públicas de manejo pode causar diversos impactos na vegetação destes parques (Rosa \& Schiavini 2006). O intenso pisoteio por parte dos visitantes em alguns locais da vegetação natural, a compactação do solo nas áreas mais frequentadas e a presença de grandes clareiras são impactos que podem influenciar significativamente a estrutura e composição vegetal, principalmente no sentido de comprometer o recrutamento e o estabelecimento dos indivíduos que irão compor a floresta no futuro. A partir do conhecimento sobre as consequências destes distúrbios e a composição e estrutura destes remanescentes florestais pode-se construir uma base teórica que subsidie sua conservação e recuperação, contribuindo substancialmente para seu manejo (Custódio Filho et al. 1994).

Um crescente número de estudos tem demonstrado que a classificação de espécies de plantas terrestres com base em suas características funcionais, como quanto ao grupo sucessional e à síndrome de dispersão, é um caminho promissor para resolver importantes questões ecológicas na escala dos ecossistemas, paisagens e biomas (Woodward \& Diament 1991). Estas questões incluem as respostas da vegetação a variações ambientais e regimes de perturbação (Cornelissen et al. 2003). $\mathrm{O}$ grande número de variáveis ambientais e a grande diversidade de estratégias apresentadas por cada espécie reforçam a importância de trabalhos descritivos que possam facilitar a compreensão do papel ecológico destas comunidades e direcionar a realização de futuros estudos (Oliveira Filho et al. 1994).

O presente trabalho teve como objetivo o levantamento fitossociológico do estrato arbóreo de um fragmento de Floresta Estacional Semidecidual (FES) localizado no Parque da Matinha, em Monte Carmelo, Minas Gerais. Por meio da caracterização sucessional das espécies amostradas e a comparação com outros remanescentes da região, este estudo também objetivou aferir sobre o estádio de conservação desta comunidade, bem como, contribuir para o conhecimento da flora do Alto Paranaíba.

\section{Material e Métodos}

Área de estudo e histórico de ocupação

O estudo foi realizado no Parque Municipal da Matinha, localizado a menos de $3 \mathrm{~km}$ a sudoeste do centro da cidade de Monte Carmelo, mesorregião do Alto Paranaíba, Minas Gerais. O fragmento florestal, cuja área é de aproximadamente 120 ha, possui a coordenada central em $18^{\circ} 45^{\prime} 02^{\prime \prime S}$ e 4730'35'W. Seguindo a classificação de Veloso et al. (1991), o fragmento possui um domínio fitogeográfico de Floresta Estacional Semidecidual. Foram percebidos indícios de perturbação antrópica como trilhas, corte seletivo de árvores e lixo urbano. 
A área está situada na Bacia Hidrográfica do rio Paranaíba, apresentando Latossolo VermelhoEscuro distrófico e altitude média de $902 \mathrm{~m}$. A partir dos valores de precipitação e temperatura disponibilizados pela Estação Meteorológica de Monte Carmelo, a região é caracterizada por apresentar um clima sazonal, com duas estações bem definidas, uma com verão quente e chuvoso, e outra com inverno frio e seco. A temperatura e o índice pluviométrico médios anuais são de $20,7^{\circ} \mathrm{C}$, e $1569,1 \mathrm{~mm}$, respectivamente. Esses valores condizem com o clima do tipo Aw, segundo o sistema de Köppen (1948).

\section{Parâmetros fitossociológicos \\ e de diversidade}

Para o delineamento amostral procurou-se fazer o levantamento em uma região nuclear do remanescente florestal, evitando gradientes com outras fisionomias e as áreas muito antropizadas. Foram demarcadas 25 parcelas permanentes (20 $\times 20 \mathrm{~m}$ cada), totalizando 1 hectare amostral (Mueller-Dombois \& Ellenberg 1974), a fim de realizar acompanhamentos futuros desta comunidade vegetal. Para a análise da vegetação arbórea, todos os indivíduos vivos com CAP (circunferência à altura do peito a uma altura de $1,30 \mathrm{~m}$ do solo) $\geq 15 \mathrm{~cm}$ foram amostrados, sendo identificada a espécie e quantificada a CAP. Indivíduos com ramificação abaixo do ponto de medida tiveram seus ramos medidos separadamente e, após o cálculo da área basal de cada ramificação, os valores foram somados para compor a medida do indivíduo.

Os parâmetros fitossociológicos como densidade, frequência, dominância e valor de importância relativos foram apresentados para todas as espécies e o índice de diversidade de Shannon (H') e equabilidade de Pielou (J') (Brower et al. 1998) para a comunidade como um todo, conforme Muller-Dombois e Ellenberg (1974). Estes atributos foram calculados por meio do programa FITOPAC 1.5 (Shepherd 2004).

Para todas as espécies amostradas foram coletados ramos férteis e/ou estéreis para posterior identificação. O material botânico em estágio fértil foi processado conforme metodologia usual e depois tombado no acervo do Herbarium Uberlandensis (HUFU) da Universidade Federal de Uberlândia e o material vegetativo testemunho de todas as espécies foi depositado no Laboratório de Ecologia Vegetal da Universidade Federal de Uberlândia. A identificação taxonômica foi feita por meio de literatura especializada, consultas ao herbário e especialistas. A partir dos dados obtidos durante o levantamento florístico foi elaborada a lista de famílias e espécies encontradas na área, segundo o sistema APG III (2009). O presente trabalho teve sua etapa de campo realizada durante o período de outubro a dezembro de 2008.

\section{Grupos sucessionais e síndrome} de dispersão

As espécies foram classificadas em grupos sucessionais, baseando-se nos trabalhos realizados por Gandolfi et al. (1995), Tabarelli \& Mantovani (1997), Pinard et al. (1999), Fonseca \& Rodrigues (2000), Silva et al. (2003), Paula et al. (2004), Santos et al. (2004) e Catharino et al. (2006). Quando houve divergências na classificação entre os trabalhos, seguiu-se a concordância da maioria destes. Assim, as espécies foram distribuídas em três grupos, seguindo a classificação de Gandolfi et al. (1995): pioneiras (P) - dependentes de luz que não ocorrem no sub-bosque, desenvolvendose em clareiras ou nas bordas da floresta; secundárias iniciais (SI) - ocorrem em condições de sombreamento médio ou luminosidade não muito intensa, ocorrendo em clareiras pequenas, bordas de clareiras grandes e bordas de floresta e secundárias tardias (ST) - desenvolve-se no subbosque em condições de sombra leve ou densa e crescem até alcançar o dossel ou a condição de emergente.

As espécies também foram classificadas quanto à síndrome de dispersão, adotando os critérios morfológicos dos frutos, definidos por van der Pijl (1982), e com auxílio de literatura (Pinheiro \& Ribeiro 2001). Dessa forma, foram classificadas em três grupos: anemocóricas (dispersão por vento), autocóricas (dispersão por gravidade e/ou explosiva) e zoocóricas (dispersão por animais).

\section{Resultados e Discussão}

Parâmetros fitossociológicos

e de diversidade

Foram amostrados 798 indivíduos, distribuídos em 98 espécies, 79 gêneros e 37 famílias (Tab. 1). O índice de diversidade de Shannon-Wiener (H') e o valor de equabilidade de Pielou (J') da comunidade foram, respectivamente, 3,97 e 0,87 . Os valores de H'e J' obtidos neste 
Tabela 1 - Espécies arbóreas $(\mathrm{CAP} \geq 15 \mathrm{~cm})$ amostradas na Floresta Estacional Semidecidual do fragmento urbano em Monte Carmelo, Minas Gerais, e suas caracterizações quanto ao grupo ecológico (sucessional e síndrome de dispersão) e valores dos parâmetros fitossociológicos. $\mathrm{NI}=$ número de indivíduos; $\mathrm{AB}=$ área basal $\left(\mathrm{m}^{2}\right) ; \mathrm{DR}=$ densidade relativa; $\mathrm{FR}=$ frequência relativa; $\mathrm{DoR}=$ dominância relativa; $\mathrm{VI}=$ valor de importância; $\mathrm{GS}=$ grupo sucessional; $\mathrm{P}=$ pioneira; $\mathrm{SI}=$ secundária inicial; $\mathrm{ST}=$ secundária tardia; $\mathrm{SD}=$ síndrome de dispersão; Zoo= zoocórica; $\mathrm{Ane}=$ anemocórica; Auto= autocórica; s/clas= sem classificação.

Table 1 - Tree species $(\mathrm{CAP} \geq 15 \mathrm{~cm})$ sampled in semideciduous seasonal forest fragment urban in Monte Carmelo, Minas Gerais, and their ecological characterizations about the ecological group (successional and dispersion syndrome) and values of the phytosociological parameters. $\mathrm{NI}=$ number of individuals; $\mathrm{AB}=$ basal area $\left(\mathrm{m}^{2}\right) ; \mathrm{DR}=$ relative density; $\mathrm{FR}=$ relative frequency; $\mathrm{DoR}=$ relative dominance; $\mathrm{VI}=$ value of importance; $\mathrm{GS}=$ successional group; $\mathrm{P}=$ pioneer; $\mathrm{SI}=$ secondary home; $\mathrm{ST}=$ secondary late; $\mathrm{SD}=$ dispersion syndrome; $\mathrm{Zoo}=$ zoochoric; $\mathrm{Ane}=$ anemochoric ; Auto $=$ autochoric; $\mathrm{s} / \mathrm{clas}=$ without classification.

\begin{tabular}{|c|c|c|c|c|c|c|c|c|c|}
\hline Espécie & Família & NI & $\mathbf{A B}$ & DR & FR & DoR & VI & GS & SD \\
\hline Copaifera langsdorffii Desf. & Fabaceae & 17 & 4,44 & 2,13 & 2,20 & 16,84 & 21,17 & ST & Zoo \\
\hline Protium heptaphyllum (Aubl.) Marchand & Burseraceae & 58 & 2,52 & 7,27 & 3,61 & 9,56 & 20,43 & ST & Zoo \\
\hline Myracrodruon urundeuva Allemão & Anacardiaceae & 28 & 2,65 & 3,51 & 2,81 & 10,05 & 16,36 & ST & Ane \\
\hline Terminalia glabrescens Mart. & Combretaceae & 35 & 1,90 & 4,39 & 4,21 & 7,21 & 15,80 & SI & Ane \\
\hline Cheiloclinium cognatum (Miers.) A.C.Sm. & Celastraceae & 45 & 1,13 & 5,64 & 4,01 & 4,27 & 13,91 & SI & Zoo \\
\hline Hirtella glandulosa Spreng. & Chrysobalanaceae & 31 & 1,68 & 3,88 & 2,81 & 6,38 & 13,07 & SI & Zoo \\
\hline Chrysophyllum marginatum (Hook. \& Arn.) Radlk. & Sapotaceae & 41 & 0,73 & 5,14 & 2,81 & 2,79 & 10,73 & $\mathrm{P}$ & Zoo \\
\hline Tapirira obtusa (Benth.) J.D.Mitch. & Anacardiaceae & 27 & 0,73 & 3,38 & 3,01 & 2,75 & 9,14 & SI & Zoo \\
\hline Virola sebifera Aubl. & Myristicaceae & 24 & 0,41 & 3,01 & 2,81 & 1,57 & 7,38 & $\mathrm{P}$ & Zoo \\
\hline Maytenus floribunda Reissek & Celastraceae & 25 & 0,14 & 3,13 & 3,41 & 0,51 & 7,05 & $\mathrm{ST}$ & Zoo \\
\hline Heisteria ovata Benth. & Olacaceae & 19 & 0,49 & 2,38 & 2,40 & 1,87 & 6,66 & SI & Zoo \\
\hline Pera glabrata (Schott) Poepp. ex Baill. & Euphorbiaceae & 11 & 0,91 & 1,38 & 1,60 & 3,45 & 6,43 & $\mathrm{P}$ & Auto \\
\hline Cordiera sessilis (Vell.) Kuntze & Rubiaceae & 25 & 0,08 & 3,13 & 2,81 & 0,32 & 6,26 & SI & Zoo \\
\hline Terminalia phaeocarpa Eichler & Combretaceae & 9 & 0,90 & 1,13 & 1,60 & 3,42 & 6,15 & ST & Ane \\
\hline Callisthene major Mart. & Vochysiacaeae & 9 & 1,16 & 1,13 & 0,60 & 4,39 & 6,12 & SI & Ane \\
\hline Pouteria torta (Mart.) Radlk. & Sapotaceae & 12 & 0,73 & 1,50 & 1,40 & 2,77 & 5,68 & SI & Zoo \\
\hline Hirtella gracilipes (Hook.f.) Prance & Chrysobalanaceae & 21 & 0,19 & 2,63 & 2,00 & 0,71 & 5,35 & SI & Zoo \\
\hline Xylopia brasiliensis Spreng. & Annonaceae & 19 & 0,12 & 2,38 & 2,40 & 0,44 & 5,23 & ST & Zoo \\
\hline Chrysophyllum gonocarpum (Mart. \& Eichler) Engl. & Sapotaceae & 21 & 0,11 & 2,63 & 2,00 & 0,42 & 5,06 & ST & Zoo \\
\hline Trichilia elegans A.Juss. & Meliaceae & 20 & 0,14 & 2,51 & 1,80 & 0,53 & 4,84 & ST & Zoo \\
\hline Guettarda viburnoides Cham. \& Schltdl. & Rubiaceae & 12 & 0,29 & 1,50 & 2,00 & 1,09 & 4,59 & SI & Zoo \\
\hline Guazuma ulmifolia Lam. & Malvaceae & 16 & 0,13 & 2,01 & 2,00 & 0,50 & 4,51 & $\mathrm{P}$ & Auto \\
\hline Diospyros hispida A.DC. & Ebenaceae & 7 & 0,51 & 0,88 & 1,40 & 1,92 & 4,20 & SI & Zoo \\
\hline Siparuna guianensis Aubl. & Siparunaceae & 14 & 0,05 & 1,75 & 2,00 & 0,17 & 3,93 & SI & Zoo \\
\hline Ixora brevifolia Benth. & Rubiaceae & 12 & 0,10 & 1,50 & 2,00 & 0,38 & 3,89 & ST & Zoo \\
\hline Tapirira guianensis Aubl. & Anacardiaceae & 10 & 0,28 & 1,25 & 1,20 & 1,08 & 3,53 & SI & Zoo \\
\hline Matayba guianensis Aubl. & Sapindaceae & 11 & 0,10 & 1,38 & 1,40 & 0,39 & 3,17 & SI & Zoo \\
\hline Aspidosperma cylindrocarpon Müll.Arg. & Apocynaceae & 6 & 0,30 & 0,75 & 1,00 & 1,15 & 2,90 & $\mathrm{ST}$ & Ane \\
\hline Eriotheca candolleana (K.Schum.) A.Robyns & Malvaceae & 6 & 0,25 & 0,75 & 1,20 & 0,93 & 2,88 & SI & Ane \\
\hline Cupania vernalis Cambess. & Sapindaceae & 9 & 0,09 & 1,13 & 1,40 & 0,33 & 2,86 & SI & Zoo \\
\hline
\end{tabular}




\begin{tabular}{|c|c|c|c|c|c|c|c|c|c|}
\hline Espécie & Família & NI & AB & DR & FR & DoR & VI & GS & SD \\
\hline Ocotea corymbosa (Meisn.) Mez & Lauraceae & 5 & 0,33 & 0,63 & 1,00 & 1,24 & 2,86 & ST & Zoo \\
\hline Cecropia pachystachya Trécul & Urticaceae & 9 & 0,12 & 1,13 & 1,20 & 0,44 & 2,77 & $\mathrm{P}$ & Zoo \\
\hline Dendropanax cuneatus (DC.) Decne. \& Planch. & Araliaceae & 9 & 0,12 & 1,13 & 1,20 & 0,44 & 2,77 & SI & Zoo \\
\hline Apuleia leiocarpa (Vogel) J.F.Macbr. & Fabaceae & 7 & 0,26 & 0,88 & 0,80 & 0,97 & 2,65 & SI & Ane \\
\hline Casearia sylvestris $\mathrm{Sw}$. & Salicaceae & 8 & 0,05 & 1,00 & 1,40 & 0,20 & 2,61 & SI & Zoo \\
\hline Luehea grandiflora Mart. \& Zucc. & Malvaceae & 7 & 0,04 & 0,88 & 1,40 & 0,17 & 2,45 & $\mathrm{P}$ & Ane \\
\hline Schefflera morototoni (Aubl.) Maguire et al. & Araliaceae & 5 & 0,21 & 0,63 & 1,00 & 0,79 & 2,42 & $\mathrm{P}$ & Zoo \\
\hline Byrsonima laxiflora Griseb. & Malpiguiaceae & 8 & 0,04 & 1,00 & 1,20 & 0,17 & 2,37 & SI & Zoo \\
\hline Pouteria gardneri (Mart. \& Miq.) Baehni & Sapotaceae & 7 & 0,12 & 0,88 & 1,00 & 0,47 & 2,34 & ST & Zoo \\
\hline Astronium nelsonrosae Santin & Anacardiaceae & 5 & 0,14 & 0,63 & 1,00 & 0,55 & 2,18 & ST & Ane \\
\hline Ardisia ambigua $\mathrm{Mez}$ & Myrsinaceae & 6 & 0,02 & 0,75 & 1,20 & 0,07 & 2,03 & ST & Zoo \\
\hline Nectandra cissiflora Nees & Lauraceae & 6 & 0,11 & 0,75 & 0,80 & 0,40 & 1,96 & ST & Zoo \\
\hline Trichilia catigua A. Juss. & Meliaceae & 7 & 0,02 & 0,88 & 1,00 & 0,07 & 1,95 & SI & Zoo \\
\hline Tabebuia roseoalba (Ridl.) Sandwith & Bignoniaceae & 5 & 0,06 & 0,63 & 1,00 & 0,21 & 1,84 & $\mathrm{P}$ & Ane \\
\hline Anadenanthera colubrina (Vell.) Brenan & Fabaceae & 6 & 0,06 & 0,75 & 0,80 & 0,23 & 1,78 & SI & Auto \\
\hline $\begin{array}{l}\text { Pseudobombax tomentosum (Mart. \& Zucc.) } \\
\text { A.Robyns }\end{array}$ & Malvaceae & 2 & 0,27 & 0,25 & 0,40 & 1,04 & 1,69 & SI & Ane \\
\hline Xylopia aromatica (Lam.) Mart. & Annonaceae & 4 & 0,03 & 0,50 & 0,80 & 0,11 & 1,42 & $\mathrm{P}$ & Zoo \\
\hline Maytenus robusta Reissek & Celastraceae & 4 & 0,02 & 0,50 & 0,80 & 0,07 & 1,37 & ST & Zoo \\
\hline Myrsine umbellata Mart. & Myrsinaceae & 4 & 0,02 & 0,50 & 0,80 & 0,07 & 1,37 & $\mathrm{P}$ & Zoo \\
\hline Hymenaea courbaril L. & Fabaceae & 4 & 0,01 & 0,50 & 0,80 & 0,04 & 1,35 & ST & Zoo \\
\hline Platypodium elegans Vogel & Fabaceae & 4 & 0,08 & 0,50 & 0,40 & 0,29 & 1,19 & SI & Ane \\
\hline Cordia trichotoma (Vell.) Arrab. ex Steud. & Boraginaceae & 3 & 0,05 & 0,38 & 0,60 & 0,17 & 1,15 & $\mathrm{P}$ & Ane \\
\hline Simira sampaioana (Standl.) Steyerm. & Rubiaceae & 3 & 0,08 & 0,38 & 0,40 & 0,32 & 1,10 & ST & Ane \\
\hline Piptadenia gonoacantha (Mart.) J.F.Macbr. & Fabaceae & 3 & 0,03 & 0,38 & 0,60 & 0,10 & 1,08 & $\mathrm{P}$ & Auto \\
\hline Ilex cerasifolia Reissek & Aquifoliaceae & 3 & 0,01 & 0,38 & 0,60 & 0,03 & 1,01 & SI & Zoo \\
\hline Styrax camporum Pohl & Styracaceae & 3 & 0,01 & 0,38 & 0,60 & 0,04 & 1,01 & SI & Zoo \\
\hline Sweetia fruticosa Spreng. & Fabaceae & 3 & 0,06 & 0,38 & 0,40 & 0,22 & 0,99 & SI & Ane \\
\hline Ficus clusiifolia Schott & Moraceae & 2 & 0,09 & 0,25 & 0,40 & 0,34 & 0,99 & SI & Zoo \\
\hline Machaerium hirtum (Vell.) Stellfeld & Fabaceae & 3 & 0,05 & 0,38 & 0,40 & 0,19 & 0,97 & $\mathrm{P}$ & Ane \\
\hline Lithraea molleoides (Vell.) Engl. & Anacardiaceae & 2 & 0,07 & 0,25 & 0,40 & 0,27 & 0,92 & $\mathrm{P}$ & Zoo \\
\hline Margaritaria nobilis L.f. & Phyllanthaceae & 2 & 0,05 & 0,25 & 0,40 & 0,21 & 0,86 & SI & Auto \\
\hline Eugenia involucrata DC. & Myrtaceae & 3 & 0,02 & 0,38 & 0,40 & 0,07 & 0,85 & ST & Zoo \\
\hline Coussarea hydrangeifolia (Benth.) Müll.Arg. & Rubiaceae & 3 & 0,01 & 0,38 & 0,40 & 0,03 & 0,80 & SI & Zoo \\
\hline Myrcia tomentosa (Aubl.) DC. & Myrtaceae & 2 & 0,03 & 0,25 & 0,40 & 0,10 & 0,75 & $\mathrm{P}$ & Zoo \\
\hline Ficus guaranitica Chodat & Moraceae & 2 & 0,02 & 0,25 & 0,40 & 0,08 & 0,73 & SI & Zoo \\
\hline Campomanesia velutina (Cambess.) O.Berg & Myrtaceae & 2 & 0,02 & 0,25 & 0,40 & 0,06 & 0,71 & $\mathrm{P}$ & Zoo \\
\hline Roupala brasiliensis Klotzsch & Proteaceae & 2 & 0,01 & 0,25 & 0,40 & 0,04 & 0,69 & ST & Ane \\
\hline Lacistema aggregatum (P.J.Bergius) Rusby & Lacistemataceae & 2 & 0,01 & 0,25 & 0,40 & 0,03 & 0,68 & $\mathrm{P}$ & Zoo \\
\hline Matayba elaeagnoides Radlk. & Sapindaceae & 2 & 0,01 & 0,25 & 0,40 & 0,03 & 0,68 & SI & Zoo \\
\hline
\end{tabular}

Rodriguésia 63(3): 489-499. 2012 


\begin{tabular}{|c|c|c|c|c|c|c|c|c|c|}
\hline Espécie & Família & NI & $\mathbf{A B}$ & DR & FR & DoR & VI & GS & SD \\
\hline Prockia crucis P.Browne ex L. & Salicaceae & 2 & 0,01 & 0,25 & 0,40 & 0,02 & 0,67 & $\mathrm{P}$ & Zoo \\
\hline Cryptocarya aschersoniana $\mathrm{Mez}$ & Lauraceae & 1 & 0,08 & 0,13 & 0,20 & 0,31 & 0,64 & ST & Zoo \\
\hline Lafoensia densiflora Pohl & Lythraceae & 1 & 0,07 & 0,13 & 0,20 & 0,26 & 0,59 & ST & Zoo \\
\hline Apeiba tibourbou Aubl. & Malvaceae & 1 & 0,05 & 0,13 & 0,20 & 0,20 & 0,52 & $\mathrm{P}$ & Auto \\
\hline Guarea guidonia (L.) Sleumer & Meliaceae & 2 & $<0,01$ & 0,25 & 0,20 & 0,02 & 0,47 & $\mathrm{ST}$ & Zoo \\
\hline Inga vera Willd. & Fabaceae & 1 & 0,02 & 0,13 & 0,20 & 0,09 & 0,41 & SI & Zoo \\
\hline Ormosia arborea (Vell.) Harms & Fabaceae & 1 & 0,02 & 0,13 & 0,20 & 0,08 & 0,40 & SI & Zoo \\
\hline Ficus obtusiuscula (Miq.) Miq. & Moraceae & 1 & 0,02 & 0,13 & 0,20 & 0,06 & 0,39 & SI & Zoo \\
\hline Cardiopetalum calophyllum Schltdl. & Annonaceae & 1 & 0,01 & 0,13 & 0,20 & 0,05 & 0,38 & SI & Zoo \\
\hline Lamanonia ternata Vell. & Cunoniaceae & 1 & 0,01 & 0,13 & 0,20 & 0,05 & 0,37 & SI & Ane \\
\hline Xylosma prockia (Turcz.) Turcz. & Salicaceae & 1 & 0,01 & 0,13 & 0,20 & 0,04 & 0,37 & $\mathrm{P}$ & Zoo \\
\hline Aspidosperma parvifolium A.DC. & Apocynaceae & 1 & 0,01 & 0,13 & 0,20 & 0,03 & 0,36 & $\mathrm{ST}$ & Ane \\
\hline Inga sessilis (Vell.) Mart. & Fabaceae & 1 & 0,01 & 0,13 & 0,20 & 0,04 & 0,36 & SI & Zoo \\
\hline Acacia polyphylla DC. & Fabaceae & 1 & 0,01 & 0,13 & 0,20 & 0,03 & 0,35 & $\mathrm{P}$ & Auto \\
\hline Aspidosperma subincanum Mart. ex A.DC. & Apocynaceae & 1 & 0,01 & 0,13 & 0,20 & 0,03 & 0,35 & SI & Ane \\
\hline Trema micrantha (L.) Blume & Cannabaceae & 1 & 0,01 & 0,13 & 0,20 & 0,02 & 0,35 & $\mathrm{P}$ & Zoo \\
\hline Bauhinia ungulata $\mathrm{L}$. & Fabaceae & 1 & $<0,01$ & 0,13 & 0,20 & 0,01 & 0,34 & SI & Auto \\
\hline Cabralea canjerana (Vell.) Mart. & Meliaceae & 1 & $<0,01$ & 0,13 & 0,20 & 0,01 & 0,34 & $\mathrm{ST}$ & Auto \\
\hline Casearia gossypiosperma Briq. & Salicaceae & 1 & $<0,01$ & 0,13 & 0,20 & 0,01 & 0,34 & SI & Zoo \\
\hline Celtis iguanaea (Jacq.) Sarg. & Cannabaceae & 1 & 0,01 & 0,13 & 0,20 & 0,02 & 0,34 & $\mathrm{P}$ & Zoo \\
\hline Chomelia pohliana Müll.Arg. & Rubiaceae & 1 & $<0,01$ & 0,13 & 0,20 & 0,01 & 0,34 & SI & Zoo \\
\hline Ficus pertusa L.f. & Moraceae & 1 & 0,01 & 0,13 & 0,20 & 0,02 & 0,34 & $\mathrm{P}$ & Zoo \\
\hline Myrcia splendens (Sw.) DC. & Myrtaceae & 1 & $<0,01$ & 0,13 & 0,20 & 0,01 & 0,34 & $\mathrm{P}$ & Zoo \\
\hline Mytaceae 1 & Myrtaceae & 1 & $<0,01$ & 0,13 & 0,20 & 0,01 & 0,34 & $\mathrm{~s} / \mathrm{clas}$ & s/clas \\
\hline Rudgea viburnoides (Cham.) Benth. & Rubiaceae & 1 & $<0,01$ & 0,13 & 0,20 & 0,01 & 0,34 & SI & Zoo \\
\hline Siphoneugena densiflora O.Berg & Myrtaceae & 1 & $<0,01$ & 0,13 & 0,20 & 0,01 & 0,34 & ST & Zoo \\
\hline Coutarea hexandra (Jacq.) K.Schum. & Rubiaceae & 1 & $<0,01$ & 0,13 & 0,20 & 0,01 & 0,33 & SI & Ane \\
\hline Handroanthus serratifolius (Vahl) S.O. Grose & Bignoniaceae & 1 & $<0,01$ & 0,13 & 0,20 & 0,01 & 0,33 & ST & Ane \\
\hline Trichilia pallida $\mathrm{Sw}$. & Meliaceae & 1 & $<0,01$ & 0,13 & 0,20 & 0,01 & 0,33 & $\mathrm{ST}$ & Zoo \\
\hline
\end{tabular}

estudo foram superiores aos obtidos em outros fragmentos da região com predomínio de Floresta Estacional Semidecidual e com os mesmos critérios de inclusão (Rosa \& Schiavini 2006; Dias Neto et al. 2009; Gusson et al. 2009; Vale et al. 2009; Prado Júnior et al. 2010; Lopes et al. 2011; Prado Júnior et al. 2011) (Tab. 2).

O tamanho do fragmento (120 ha) e a ocorrência de algumas manchas de cerradão e floresta de galeria, além da FES neste fragmento aumentam a heterogeneidade ambiental do gradiente florestal e contribuem para o aumento da diversidade na área (Costa \& Araújo 2001). Segundo a teoria do distúrbio intermediário (Connell 1978), os impactos antrópicos como corte de árvores, trilhas internas e proximidade com a área urbana também podem influenciar a elevada diversidade florística desta comunidade vegetal, já que, durante estádio intermediário, a heterogeneidade ambiental é maior e permite 
Tabela 2 - Valores dos parâmetros da estrutura horizontal por grupo ecológico das espécies amostradas no Parque da Matinha, Monte Carmelo, Minas Gerais. $\mathrm{DR}=$ densidade relativa, $\mathrm{FR}=$ frequência relativa, DoR $=$ dominância relativa, $\mathrm{VI}=$ valor de importância.

Table 2 - Parameters values of the horizontal structure for each successional group of species sampled in "Parque da Matinha", Monte Carmelo, Minas Gerais. DR = relative density, $\mathrm{FR}=$ relative frequency, $\mathrm{DoR}=$ relative dominance, $\mathrm{VI}=$ value of importance.

\begin{tabular}{lcccc}
\hline Grupo sucessional & DR(\%) & FR(\%) & DoR(\%) & VI (\%) \\
\hline Pioneiras & 19,1 & 20,4 & 11,4 & 16,9 \\
Secundárias Iniciais & 47,2 & 47,1 & 41,4 & 45,3 \\
Secundárias Tardias & 33,8 & 32,2 & 47,2 & 37,8 \\
\hline
\end{tabular}

a co-ocorrência de espécies com diferentes características, dificultando a dominância de poucas espécies, como ocorre nos estádios iniciais e finais da sucessão florestal. $\mathrm{O}$ alto número de espécies amostradas na área (98 espécies) (Tab. 2) também contribuiu para o alto valor de equabilidade, já o aumento da diversidade tende a aumentar a uniformidade nas proporções das densidades entre as espécies da comunidade (Uhl \& Murphy 1981).

A área basal estimada de $26,4 \mathrm{~m}^{2} \cdot \mathrm{ha}^{-1}$ foi inferior à média $28,9 \mathrm{~m}^{2}$.ha ${ }^{-1}$ encontrada nos demais trabalhos comparados na Tabela 2, o que pode ser indicador de uma comunidade no estádio intermediário de sucessão. A ocorrência de corte raso na área atua diretamente na redução da área basal desta comunidade, já que cerca de $90 \%$ da biomassa das florestas tropicais está acumulada nos indivíduos de grande porte do dossel (Ozanne et al. 2003), e estes tendem a ser os mais visados em relação ao corte seletivo.

Dentre as famílias com maior riqueza de espécies destacaram-se Fabaceae (13 espécies), Rubiaceae (8 espécies) e Myrtaceae (6 espécies) (Tab. 1). Com relação à densidade de cada família, a maior abundância foi de Sapotaceae, Celastraceae e Anacardiaceae, que totalizaram 28,5\% dos indivíduos amostrados (Tab. 1). Estas famílias têm ampla distribuição, não apenas nas florestas estacionais semideciduais, mas nas florestas tropicais em geral (Oliveira-Filho \& Fontes 2000). A grande representatividade de Fabaceae e Rubiaceae pode ser um indicativo da alta perturbação na área, já que diversos estudos apontam uma tendência à redução da importância de Rubiaceae e Fabaceae em florestas com estádio sucessional avançado (Greig-Smith 1952; Tabarelli \& Mantovani 1999; Vale et al. 2009).
Myracrodruon urundeuva Allemão, popularmente conhecida como aroeira, amostrada no parque como a terceira espécie com maior VI, está na lista de espécies ameaçadas da flora de Minas Gerais (Fundação Biodiversitas 2007), sendo considerada vulnerável à extinção. Sua madeira possui alta resistência mecânica e durabilidade, apresentando densidade de 1,19 $\mathrm{g} / \mathrm{cm}^{3}$, o que potencializa sua exploração e consequentemente sua vulnerabilidade (Rizzini 1971). Deve-se salientar que o corte seletivo da aroeira para uso na indústria madeireira praticamente extinguiu os indivíduos de grande porte (Brandão 2000) e, em decorrência da intensa exploração, esta espécie vem sofrendo grande redução de suas populações naturais (Nunes et al. 2008). A presença desta espécie no fragmento justifica esforços no sentido da sua preservação, bem como de outros remanescentes florestais da região.

Segundo o Catálogo das Árvores Nativas de Minas Gerais (Oliveira-Filho 2006), esta é a primeira ocorrência de Lacistema aggregatum (P.J.Bergius) Rusby (Lacistemataceae) e Matayba elaeagnoides Radlk. para as florestas estacionais semideciduais do Estado de Minas Gerais.

\section{Grupos sucessionais e síndromes de dispersão}

A Tabela 1 mostra a classificação quanto aos grupos sucessionais e às síndromes de dispersão das espécies amostradas. Foram classificadas 25 espécies pioneiras ( $26 \%$ esp., $19 \%$ ind.), 44 secundárias iniciais (45\% esp., $47 \%$ ind.) e 28 secundárias tardias ( $29 \%$ esp., $34 \%$ ind.). A redução na porcentagem de indivíduos pioneiros em relação às espécies pioneiras está relacionada à 
baixa densidade da maioria das mesmas, sendo que 17 das 25 espécies pioneiras estão representadas por menos de cinco indivíduos. Budowski (1970) considera que o estádio sucessional de uma floresta é dado pelo grupo sucessional que apresentar mais de 50\% dos indivíduos. Neste estudo, os maiores valores de densidade, frequência e VI (Tab. 2) pertenceram ao grupo das secundárias iniciais, que exerceram a maior influência na estrutura florestal. Em relação à dominância, as secundárias tardias representaram os maiores valores (Tab. 2), o que pode estar relacionado ao fato destas espécies apresentarem maior longevidade e, conseqüentemente, maior incremento de área basal nas formações florestais (Silvertown \& Doust 1993). Assim, os resultados sugerem que a floresta estudada se encontra em um estádio intermediário de desenvolvimento sucessional, podendo ser considerado como um remanescente de Floresta Estacional Semidecidual secundário quanto à sua conservação.

Apesar do aparente potencial em direcionarse a um estádio sucessional mais maduro, sugerido pela maior dominância das espécies secundárias tardias, o Parque da Matinha sofre impactos que influenciam significativamente sua composição vegetal, principalmente no sentido de comprometer o recrutamento e o estabelecimento dos indivíduos que irão compor a estrutura da floresta no futuro. Comparando a área de estudo com outros fragmentos de FES da região (Dias-Neto et al. 2009; Vale et al. 2009; Prado Júnior et al. 2010), as espécies pioneiras apresentaram maior representatividade florística e estrutural. Por se tratar de um fragmento urbano, a interferência antrópica é marcada por trilhas, corte seletivo e presença de resíduos sólidos. A ausência de políticas públicas que diminuam os impactos resultantes da intensa visitação pública potencializa a perturbação do ecossistema, aumentando o número de clareiras e, consequentemente, favorecendo o desenvolvimento do grupo das espécies pioneiras.

Em relação à síndrome de dispersão pôdese observar que a amostragem apresentou uma composição de $68 \%$ espécies zoocóricas, $22 \%$ anemocóricas e $9 \%$ autocóricas. Uma proporção ainda maior foi observada quando analisados os indivíduos, sendo que os indivíduos zoocóricos representaram $77 \%$ da comunidade. Estes resultados seguem os padrões de florestas tropicais, onde mais de $50 \%$ das espécies produzem frutos adaptados ao consumo de pássaros e mamíferos (Howe \& Smallwood 1982). Muitos animais tropicais dependem parcial ou inteiramente de frutos para sua alimentação pelo menos em parte do ano (Janzen 1980). Muitas espécies zoocóricas apresentam padrão de frutificação sequencial, ou seja, produzem frutos durante todo o ano, ao contrário das espécies anemocóricas, que comumente frutificam no período seco do ano, onde o que seria mais forte nessa estação, (Morellato et al. 1989). Demonstra-se, assim, a importância deste fragmento na oferta de recursos para a fauna local, e consequentemente, no equilibrio entre os processos ecólogicos das formações florestais.

As características estruturais e florísticas deste remanescente ampliam o conhecimento sobre a flora do Alto Paranaíba e Triângulo Mineiro. A alta riqueza de espécies, abundância dos grupos de espécies secundárias iniciais e secundárias tardias, bem como a alta porcentagem de espécies zoocóricas reforçam a necessidade de criar políticas públicas para utilização do parque pelos visitantes. Tais resultados aumentam a valoração do Parque da Matinha, reafirmando a importância deste parque urbano na conservação da biodiversidade, reabilitação dos processos ecológicos e abrigo da fauna e flora nativa.

\section{Referências}

APG - Angiosperm Phylogeny Group. 2009. An update of the Angiosperm Phylogeny Group classification for the order and families of flowering plants: APG III. Botanical Journal of the Linnean Society 161: 105-121.

Aquino, F.G. \& Miranda, G.H.B. 2008. Conseqüências ambientais da fragmentação de hábitats no Cerrado. In: Sano, S.; Ribeiro, J.P. \& Almeida, S.P. (eds.). Cerrado: ecologia e flora. Embrapa Cerrados, Planaltina 1: 385-388.

Brandão, M. 2000. Caatinga. In: Mendonça, M.P.; Lins, L.V. (orgs). Lista vermelha das espécies ameaçadas de extinção da flora de Minas Gerais. Fundação Biodiversitas e Fundação Zôo-Botânica de Belo Horizonte 1: 75-85.

Brower, J.E.; Zar, J.H. \& Ende, C.N. 1998. Field and laboratory methods for general ecology. $4^{\mathrm{a}}$ ed. WCB McGraw-Hill. 273p.

Budowski, G. 1970. The distinction between old secondary and climax species in tropical central american lowland rainforest. Tropical Ecology 11: 44-48. 
Catharino, E.L.M.; Bernacci, L.C.; Franco, G.A.D.C.; Durigan, G.; Metzger, J.P. 2006. Aspectos da composição e diversidade do componente arbóreo das florestas da Reserva Florestal do Morro Grande, Cotia, SP. Biota Neotropica 6: 1-28.

Chiesura, A. 2004. The role of urban parks for the sustainable city. Landscape and Urban Planning 68: 129-138.

Coley, R.; Kuo, F. \& Sullivan, W. 1997. Where does community grow? The social context created by nature in urban public housing. Environmental Behavior 29: 468-494.

Connell, J.H. 1971. On the role of natural enemies in preventing competitive exclusion in some marine animals and in forest trees. In: Den Boer, P.J. \& Gradwell, G.R. (eds). Dynamics of populations Centre for Agricultural Publishing and Documentation, Wageningen. Pp. 298-312.

Conway, H. 2000. Parks and people: the social functions. In: Woudstra, J. \& Fieldhouse, K. (eds.). The regeneration of public parks. E\&FN Spon and The Garden History Society Landscape Design Trust 1: 9-19.

Cornelissen, J.H.C.; Lavorel, S.; Garnier, E.; Diaz, S.; Buchmann, N.; Gurvich, D.E.; Reich, P.B.; ter Steege, H.; Morgan, H.D.; van der Heijden, M.G.A.; Pausas J.G. \& Poorter, H. 2003. A handbook of protocols for standardized and easy measurement of plant functional traits worldwide. Australian Journal of Botany 51: 335-380.

Costa, A.A. \& Araújo, G.M. 2001. Comparação arbórea de cerrado e cerradão na Reserva do Panga, Uberlândia, MG. Acta Botanica Brasilica 15: 63-72.

Custódio Filho, A.; Franco, G.A.D.C. \& Dias, A.C. 1994. Composição florística de um trecho de floresta pluvial atlântica em regeneração natural após desmatamento diferenciado em Pariqüera-Açu, SP - Brasil. Revista do Instituto Florestal 6: 87-98.

Dias Neto, O.C.; Schiavini, I.; Lopes, S.F.; Vale, V.S.; Gusson, A.E. \& Oliveira, A.P. 2009. Estrutura fitossociológica e grupos ecológicos em fragmento de floresta estacional semidecidual, Uberaba, Minas Gerais, Brasil. Rodriguésia 60: 1087-1100.

Durigan, G.; Franco, G.A.D.C.; Saito, M. \& Baitello, J.B. 2000. Estrutura e diversidade do componente arbóreo da floresta na Estação Ecológica dos Caetetus, Gália, SP. Revista Brasileira de Botânica, 23: 371-383.

Dwyer, J.E.; McPherson, E.G.; Schroeder, H.W. \& Rowntree, R.A. 1992. Assessing the benefits and costs of the urban forest. Journal of Arboriculture 18: $227-234$

Fonseca, R.C.B. \& Rodrigues, R.R. 2000. Análise estrutural e aspectos do mosaico sucessional de uma floresta semidecídua em Botucatu, SP. Scientia Florestalis 57: 27-43.

Fundação Biodiversitas. 2007. Revisão das listas das espécies da flora ameaçadas de extinção do Estado de Minas Gerais. Mendonça, M.P. (coord.). Disponível em: <http://www.biodiversitas.org. br/listas-mg/lista_floramg.asp $>$. Acesso em 17 Dez 2010.

Galetti, M.; Costa C. \& Cazetta, E. 2003. Effects of forest fragmentation, anthropogenic edges and fruit color on the consumption of ornithochoric fruits. Biological Conservation 111: 269-293.

Gandolfi, S.; Leitão-Filho, H.F. \& Bezerra, C.L.F. 1995. Levantamento florístico e caráter sucessional das espécies arbustivo-arbóreas de uma floresta mesófila semidecídua no município de Guarulhos, SP. Revista Brasileira de Biologia 55: 753-767.

Greig-Smith P. 1952. Ecological observations on degraded and secondary forest in Trinidad, British West Indies: I. General features of the vegetation. Journal of Ecology 40: 283-330.

Gusson, A. E.; Lopes, S. F.; Dias Neto, O. C.; Vale, V. S.; Oliveira, A. P.; Schiavini, I. 2009. Características químicas do solo e estrutura de um fragmento de floresta estacional semidecidual em Ipiaçu, Minas Gerais, Brasil. Rodriguésia 60: 403-414.

Hartig, T.; Mang, M. \& Evans, G. 1991. Restorative effects of natural environments experiences. Environmental behavior 23: 3-26.

Howe, H.F. \& Smallwood, J. 1982. Ecology of seed dispersal. Annual Review of Ecology, Evolution and Systematics 13: 201-228.

Janzen, D. 1980. Ecologia vegetal nos trópicos. EPU, São Paulo. 79p.

Koeppen, W. 1948. Climatologia: con un estudio de los climas de la tierra. Fondo de Cultura Economica, Ciudad de México. 478p.

Lopes, S.F.; Schiavini, I.; Prado Júnior, J.A.; Gusson, A.E.; Souza Neto, A.R.; Vale, V.S. \& Dias Neto, O.C. 2011. Caracterização ecológica e distribuição diamétrica da vegetação arbórea em um remanescente de Floresta Estacional Semidecidual, na Fazenda Experimental do Glória, Uberlândia, MG. Bioscience Journal 27: 322-335.

Morellato, L.P.C., Rodrigues, R.R., Leitão-Filho, H.F. \& Joly C.A. 1989. Estudo comparativo da fenologia de espécies arbóreas de floresta de altitude e floresta mesófila semidecídua na Serra do Japi, Jundiaí, São Paulo. Revista Brasileira de Botânica 12: 85-98.

Mueller-Dombois, D. \& Ellenberg, H. 1974. Aims and methods of vegetation ecology. John Wiley \& Sons, New York. 547p.

Nowak, D.J.; Crane, D.E. \& Stevens, J.C. 2006. Air pollution removal by urban trees and shrubs in the 
United States. Urban Forestry and Urban Greening 4: 115-123.

Nunes, Y.R.F.; Fagundes, M.; Almeida, H.S. \& Veloso, M.D.M. 2008. Aspectos ecológicos da aroeira (Myracrodruon urundeuva AllemãoAnacardiaceae): fenologia e germinação de sementes. Revista Árvore 32: 233-243.

Oliveira-Filho, A.T. 2006. Catálogo das árvores nativas de Minas Gerais: mapeamento e inventário da flora nativa e dos reflorestamentos de Minas Gerais. Ed. UFLA, Lavras. 423p.

Oliveira-Filho, A.T.; Vilela E.A.; Carvalho, D.A. \& Gavilanes, M.L. 1994. Differentiation of streamside and upland vegetation in area of montane semidecidous forest in southeartern Brazil. Flora 189: 287-305.

Ozanne, C.M.P.; Anhuf, D.; Boulter, S.L.; Keller, M.; Kitching, R.L.; Korner, C.; Meinzer, F.C.; Mitchell, A.W.; Nakashizuka. T.; Dias. P.L.S.; Stork. N.E.; Wright. S.J.\& Yoshimura M. 2003. Biodiversity meets the atmosphere: A global view of forest canopies. Science 301: 183-186.

Paula, A.; Silva, A.F.; Marco-Júnior, P.; Santos, F.A.M. \& Souza, A.L. 2004. Sucessão ecológica da vegetação arbórea em uma floresta estacional semidecidual, Viçosa, MG, Brasil. Acta Botanica Brasilica 18: 407-423.

Pimm, S.L.; Ayres, M.; Balmford, A.; Branch, G.; Brandon, K.; Brooks, T.; Bustamante, R.; Costanza, R.; Cowling, R.; Curran, L.M.; Dobson, A.; Farber, S,; Fonseca, G.A.B.; Gascon, C.; Kitching, R.; McNeely, J.; Lovejoy, T.; Mittermeier, R.A.; Myers, N.; Patz, J.A.; Raff, B.; Rapport, D.; Raven, P.; Roberts, C.; Rodríguez, J.P.; Rylands, A.B.; Tucker, C.; Safina, C.; Samper, C.; Stiassny, M.L.J.; Supriatna, J.; Wall, D.H. \& Wilcove, D. 2001. Can we defy nature's end? Science 293: 2207-2208.

Pinard, M.; Putz, F.; Rum, Z.D.; Guzmán, R. \& Jardim, A. 1999. Ecological characterization of tree species for guiding forest management decisions in seasonally dry forests in Lomério, Bolivia. Forest Ecology and Management 113: 201-213.

Pinheiro, F. \& Ribeiro, J.F. 2001. Síndromes de dispersão de sementes em Matas de Galeria do Distrito Federal. In: Ribeiro, J.F.; Fonseca, C.E.L. \& Souza-Silva, J.C. (eds.). Cerrado: caracterização e recuperação de Matas de Galeria. Vol. 1. Embrapa Cerrados, Planaltina. Pp. 335-378.

Prado Júnior, J.A.; Vale, V.S.; Oliveira, A.; Gusson, A.E.; Dias Neto, O.C.; Lopes, S.F. \& Schiavini, I. 2010. Estrutura da comunidade arbórea em um fragmento de floresta estacional semidecidual localizada na reserva legal da Fazenda Irara, Uberlândia, MG. Bioscience Journal 26: 638-647.
Prado Júnior, J.A.; Lopes, S.F.; Vale, V.S.; Oliveira, A.; Gusson, A.E.; Dias Neto, O.C. \& Schiavini, I. 2011. Estrutura e caracterização sucessional da comunidade arbórea de um remanescente de floresta estacional semidecidual, Uberlândia, MG. Caminhos de Geografia 12: 81-93.

Rizzini, C.T. 1971. Árvores e madeiras úteis do Brasil: manual de dendrometria brasileira. Edgard Bliicher/EDUSP, São Paulo. 294p.

Rosa, A.G. \& Schiavini, I. 2006. Estrutura da comunidade arbórea em um remanescente florestal urbano (Parque do Sabiá, Uberlândia, MG). Bioscience Journal 22: 151-162.

Sander, H.; Polasky, S. \& Haight, R.G. 2010. The value of urban tree cover: A hedonic property price model in Ramsey and Dakota Counties, Minnesota, USA. Ecological Economics 69: 1646-1656

Santos, J.H.S.; Ferreira, R.L.C.; Silva, J.A.A.; Souza, A.L.; Santos, E.S. \& Meunier, I.M.J. 2004. Distinção de grupos ecológicos de espécies florestais por meio de técnicas multivariadas. Revista Árvore 28: 387-396.

Sherperd, G.J. 2004. FITOPAC 1.5: Manual do usuário. Departamento de Botânica, UNICAMP. 96p.

Silva, F.V.; Venturin, N.; Oliveira-Filho, A.T.; Macedo, R.L.G.; Carvalho, W.A.C. \& van den Berg, E. 2003. Caracterização estrutural de um fragmento de floresta semidecídua no município de Ibiturama, MG. Cerne 9: 92-106.

Silvertown, J.W. \& Doust, J.L. 1993. Introduction to plant population biology. Blackwell Science, London. 209p.

Tabarelli, M. \& Mantovani, W. 1997. Colonização de clareiras naturais na floresta atlântica no sudeste do Brasil. Revista Brasileira de Botânica 20: 57-66.

Tabarelli, M.; Mantovani. W. \& Peres, C.A. 1999. Effects of habitat fragmentation on plant guild structure in the montane Atlantic forest of southeastern Brazil. Biological Conservation 91: 119-127.

Tagtow, R. 1990. The need for urban forests. American City County 105: 74-75.

Uhl, C. \& Murphy, P. G. 1981. Composition, structure, and regeneration of a "tierra firme" forest in the Amazon Basin of Venezuela. Tropical Ecology 22: 219-237.

Ulrich, R.S. 1981. Natural versus urban sciences: some psycho-physiological effects. Environmental Behavior 13: 523-556.

Vale, V.S.; Schiavini, I.; Lopes, S.F.; Dias Neto, O.C.; Oliveira, A. \& Gusson, A.E. 2009. Composição florística e estrutura do componente arbóreo em um remanescente primário de floresta estacional 
semidecidual em Araguari, Minas Gerais, Brasil. Hoehnea 36: 417-429.

van der Pijl, L. 1982. Principles of dispersal in higher plants. $2^{\circ}$ ed. Springer-Verlag, Berlin.

Veloso, H.; Rangel Filho, A. \& Lima, J. 1991.

Classificação da vegetação brasileira, adaptada a um sistema universal. Ministério da Economia, Brasília. 123p.

Woodward, F.I. \& Diament, A.D. 1991. Functional approaches to predicting the ecological effects of global change. Functional Ecology 5: 202212 . 